\title{
Nonlinear Disturbance Observer based Sliding Mode Control for a Class of Uncertain Nonaffine Nonlinear Systems
}

\author{
Zhongjuan $\mathrm{Li}^{1,}$, , Lingling Yang ${ }^{1, \mathrm{~b}}$ \\ ${ }^{1}$ School of Automation, Guangdong University of Technology, Guangzhou, 510006, China \\ aemail: lizhongjuan@126.com, bemail:505049@163.com
}

\begin{abstract}
Keywords: Nonaffine Nonlinear Systems; Nonlinear Disturbance Observer; Sliding Mode Control; Taylor Expansion;
\end{abstract}

\begin{abstract}
In this paper, the sliding mode tracking control is proposed for a class of uncertain non-affine nonlinear systems via the nonlinear disturbance observer(NDOB). Based on the Taylor expansion method, the affine system is approximated to facilitate the desired control design. Then, the NDOB is adopted to estimate the unknown disturbance. Subsequently, based on approximated affine nonlinear model and the NDOB, the sliding mode tracking control is proposed for non-affine nonlinear systems with uncertainty. The control scheme can guarantee semi-global uniform boundedness of the closed-loop system signals as proved by Lyapunov analysis. Numerical simulation results are presented to illustrate the effectiveness of the proposed sliding mode tracking control scheme.
\end{abstract}

\section{Introduction}

In practice, there are many nonlinear systems with nonaffine structure, which has no affine appearance of control input. It is a difficult task to design a stable controller for nonaffine nonlinear systems since the control inputs impact the dynamic behavior of the systems through a nonlinear and implicit way.

Numerous attempts have been made in the literature on controller design for nonaffine nonlinear systems. The mean-value theorem and the implicit function theorem are used to generate an affine system[1]. Taylor series expansion is exploited to get an affine form of system[2]. With their universal approximation capabilities, neural networks and fuzzy systems are adopted to establish an affine model of system[3].

It is well known that disturbances widely exist in practical systems, which includes external disturbances, unmodelled dynamics and parameter perburbations. Disturbance observer(DOB) technique was originally presented by Ohnish in 1987[4]. A nonlinear disturbance observer(NDOB) was proposed by Chen, and employed in robotic manipulators, missiles, helicopters and nonlinear systems. The DOB-based controllers have been widely studied due to their transparency in controller design and excellent disturbances attenuation ability[5] .

Sliding mode control(SMC) is a well-known efficient control scheme for nonlinear systems with uncertainties. NDOB based SMC approach is proposed for nonlinear systems with mismatched uncertainties[6]. Where, the first derivatives of the mismatched uncertainties are assumed to go to zero in the steady state. Ginoya extends the results in [6] by proposing an extended DOB, a modified sliding surface and a modified control. Where, the mismatched disturbances and their first derivatives are assumed to be bounded[7]. Xiao presents the output feedback SMC method based on DOB with the same assumption as above[8]. But all these results are applied for the affine nonlinear systems. Zhang presents integral terminal SMC scheme for nonaffine nonlinear systems[2].

This paper is to develop a sliding mode tracking control for a class of uncertain nonaffine nonlinear systems. Firstly, Taylor expansion method is applied to get the affine form of the nonlinear systems. Then, the unmeasured disturbances are estimated by the NDOB, and the estimation is used to design the sliding mode controller. Semiglobal uniform boundedness stability is proved using Lyapunov approach with the assumption that the disturbances and their first derivatives are bounded. Finally, the control performance is illustrated by simulation example. 


\section{Problem Formulation and Preliminaries}

Consider the following second-order nonaffine nonlinear system with external disturbance[1]

$\left\{\begin{array}{l}\dot{x}_{1}=x_{2} \\ \dot{x}_{2}=f(x, u)+d(t)\end{array}\right.$

where $x_{1}$ and $x_{2}$ are states, $u$ is the control input. The nonlinear function $f(x, u)$ is known and sufficiently smooth, and $d(t)$ is the external disturbance.

The control objective is to ensure closed-loop asymptotical tracking performance in the presence of time-varying external disturbance. let us consider the following assumptions and lemmas.

Assumption 1: For all $t>0$, there exist $\Delta_{i}>0$ such that the reference signal $x_{d}$ satisfies $\left|x_{d}^{(i)}(t)\right| \leq \Delta_{i}, \quad i=1,2$.

Lemma 1: For bounded initial conditions, if there exists a continuous and positive definite Lyapunov function $V(x)$ satisfying $\pi_{1}(\|x\|) \leq V(x) \leq \pi_{2}(\|x\|)$ such that $\dot{V}(x) \leq-c_{1} V(x)+c_{2}$, where $\pi_{1}, \pi_{2}$ are class $K$ functions and $c_{1}, c_{2}$ are positive constants, then the solution $x(t)$ is uniformly bounded.

Assumption 2: Consider $\|u\| \in[0, \delta]$ and $0 \leq\|\partial f / \partial u\| \leq \breve{\delta}$, where $\delta$ and $\breve{\delta}$ are two finite positive constants.

Assumption 3: For the system unknown external disturbance $d(t)$, there exist unknown positive constant $d_{M}$ and $\alpha$, such that $\forall t \in R^{+}$satisfy $|d(t)| \leq d_{M}$ and $|\dot{d}(t)| \leq \alpha$.

\section{Main Results}

(A) Taylor expansion method

Let $x=\left[x_{1}, x_{2}\right]^{T}$, then system (1) can be expressed as

$\dot{x}=f_{1}(x, u)+g_{2} d$

where $f_{1}(x, u)=\left[x_{2}, f(x, u)\right]^{T}$ and $g_{2}=[0,1]^{T}$.

Using the novel Taylor expansion method introduced by [2], system (2) yields

$\dot{x}=F(x, v)+g_{1}(x, v) u+O(\cdot)+g_{2} d$

where $\quad \dot{v}=-k_{v} v+k_{v} u \quad, \quad F(x, v)=\left[x_{2}, f_{2}(x, v)\right]^{T} \quad, \quad g_{1}(x, v)=[0, g(x, v)]^{T}$

$f_{2}(x, v)=f_{1}(x, v)-g(x, v) v, \quad g(x, v)=\left.\left(\partial f_{1}(x, u) / \partial u\right)\right|_{u=v}, \quad$ the remainder $O(\cdot)=\left[0, O_{2}(\cdot)\right]^{T}$,

$O_{2}(\cdot)=(u-v)^{2} f_{d d} / 2$ is bounded by $\left\|O_{2}(\cdot)\right\| \leq r_{p}\|u-v\|^{2} / 2$, where $f_{d d}=\left.\left(\partial^{2} f(x, u) / \partial u^{2}\right)\right|_{u=\xi}$, $\xi$ is a point between $u$ and $v, 0 \leq\left\|f_{d d}\right\| \leq r_{p}$ with $r_{p}$ as a finite positive number.

(B) NDOB design

Considering the remainder $O(\cdot)$ will be controlled by the robust term to be designed later, we design the NDOB for the following system.

$\dot{x}=F(x, v)+g_{1}(x, v) u+g_{2} d$

The NDOB is introduced and depicted by [6]

$\left\{\begin{array}{l}\dot{p}=-l g_{2} p-l\left[g_{2} l x+F(x, v)+g_{1}(x, v) u\right] \\ \hat{d}=p+l x\end{array}\right.$

where $\hat{d}, p$ and $l$ are the estimation of the disturbance, the internal state of the nonlinear observer, the observer gain to be designed, respectively.

Define the estimation error $\tilde{d}=d-\hat{d}$. The derivative of $\hat{d}$ is given as 
$\dot{\hat{d}}=\lg _{2} \tilde{d}$

Considering the definition of $\tilde{d}$, we can get

$\dot{\tilde{d}}=\dot{d}-\lg _{2} \tilde{d}$

(C) Sliding mode tracking control

Define the sliding-mode surface as

$s=\lambda_{1} e_{1}+e_{2}$

where the tracking error $e_{1}=x_{1}-x_{d}, e_{2}=x_{2}-\dot{x}_{d}, \lambda_{1}>0$ is a control parameter to be designed.

We consider the following control vector $u$ as

$u=-g(x, v)^{-1}\left[\lambda_{1} e_{2}-\ddot{x}_{d}+f_{2}(x, v)+\hat{d}+k s+\gamma_{s}\right]$

where $k>0$ is a design parameter, $\hat{d}$ is defined in (5). To restrain the dynamic error $\mathrm{O}_{2}(\cdot)$ from (3), the robust term $\gamma_{s}$ is designed as[2]

$$
\gamma_{s}=\left\{\begin{array}{l}
\frac{\varsigma s}{\|s\|}, \quad\|s\| \neq 0 \\
0, \quad s=0
\end{array}\right.
$$

where $\varsigma>\left\|O_{2}(\cdot)\right\|$ is a design constant.

(D) Stability analysis

Theorem 1: Considering the uncertain nonaffine nonlinear system (1) with the unknown external disturbance, affine nonlinear approximation by Taylor expansion is given as (3) and the NDOB is designed as (5). If the proposed sliding mode tracking control and the robust term are chosen as (9) and (10), then all signals of the closed-loop system are semiglobally uniformly stable.

Proof: Taking the derivative of the sliding surface $s$ defined in (8) along system (3), yields

$\dot{s}=\lambda_{1} e_{2}-\ddot{x}_{d}+f_{2}(x, v)+g(x, v) u+d+O_{2}(\cdot)$

Substituting the control law (9) into (11), yields

$\dot{s}=-k s+\tilde{d}-\gamma_{s}+O_{2}(\cdot)$

Consider the Lyapunov function candidate

$V=\frac{1}{2} s^{2}+\frac{1}{2} \tilde{d}^{2}$

Considering (7) and (12), the time derivative of $V$ along the state trajectory is

$$
\begin{aligned}
\dot{V} & =s \dot{s}+\tilde{d} \dot{\tilde{d}} \\
& =-k s^{2}+\tilde{d} s-\gamma_{s} s+O_{2}(\cdot) s+\tilde{d} \dot{d}-\lg _{2} \tilde{d}^{2} \\
& \leq-k s^{2}+\frac{\tilde{d}^{2}}{2}+\frac{s^{2}}{2}-\gamma_{s} s+O_{2}(\cdot) s+\frac{\dot{d}^{2}}{2}+\frac{\tilde{d}^{2}}{2}-\lg _{2} \tilde{d}^{2} \\
& =-\left(k-\frac{1}{2}\right) s^{2}-\left(\lg _{2}-1\right) \tilde{d}^{2}+\frac{\dot{d}^{2}}{2}-\gamma_{s} s+O_{2}(\cdot) s \\
& \leq-\left(k-\frac{1}{2}\right) s^{2}-\left(\lg _{2}-1\right) \tilde{d}^{2}+\frac{\alpha^{2}}{2}-\gamma_{s} s+O_{2}(\cdot) s \\
& \leq-\left(k-\frac{1}{2}\right) s^{2}-\left(\lg _{2}-1\right) \tilde{d}^{2}+\frac{\alpha^{2}}{2}-s\|s\|+\left\|O_{2}(\cdot)\right\|\|s\| \\
& \leq-\left(k-\frac{1}{2}\right) s^{2}-\left(\lg _{2}-1\right) \tilde{d}^{2}+\frac{\alpha^{2}}{2} \\
& \leq-\rho V+\frac{\alpha^{2}}{2}
\end{aligned}
$$


where $\rho:=\min \left(2\left(k-\frac{1}{2}\right), 2\left(\lg _{2}-1\right)\right)$.

To ensure the closed-loop stability, the corresponding design parameters $k$ and $l$ should be chosen such that $k-\frac{1}{2}>0, g_{2}-1>0$. According to (14) and Lemma 1 , it can be shown that the signals $s$ and $\tilde{d}$ are semiglobally uniformly bounded.

\section{Simulation Results}

A Duffing-Holmes chaotic system with control input is used for simulation study[1]

$$
\left\{\begin{array}{l}
\dot{x}_{1}=x_{2} \\
\dot{x}_{2}=-p_{1} x_{1}-p_{2} x_{2}-x_{1}^{3}+q \cos (\omega t)+h(x, u)+d(t)
\end{array}\right.
$$

where $p_{1}=0.3+0.2 \sin (10 t), \quad q=5+0.1 \cos (t), \quad p_{2}=0.2+0.2 \cos (5 t), \quad \omega=0.5+0.1 \sin (t)$, $h(x, u)=u+0.5 \cos (u)$, and the external disturbance $d=0.4 \sin (0.2 \pi t)+0.3 \sin \left(x_{1} x_{2}\right)$.

The NDOB based sliding mode tracking control is designed as (9). The design parameters are chosen as $k_{v}=50, \lambda_{1}=10, k=10, l=\left[\begin{array}{ll}0 & 6\end{array}\right]$, and $\varsigma=0.01$. The desired trajectory is given by $x_{d}=\sin (t)+\cos (0.5 t)$. The initial states are arbitrarily chosen as $x=\left[\begin{array}{ll}0.8,0 & 0\end{array}\right]^{T}, v=0, p=0$.

From Figs. 1 and 2, we can observe that the tracking performance is satisfactory and the tracking error quickly converges to zero in the presence of the time-varying external disturbance. Fig. 4 shows the NDOB exhibits good tracking performance. Based on these simulation results, we can conclude that the proposed NDOB based sliding mode tracking control is valid for the uncertain nonaffine nonlinear system with the time-varying external disturbance.

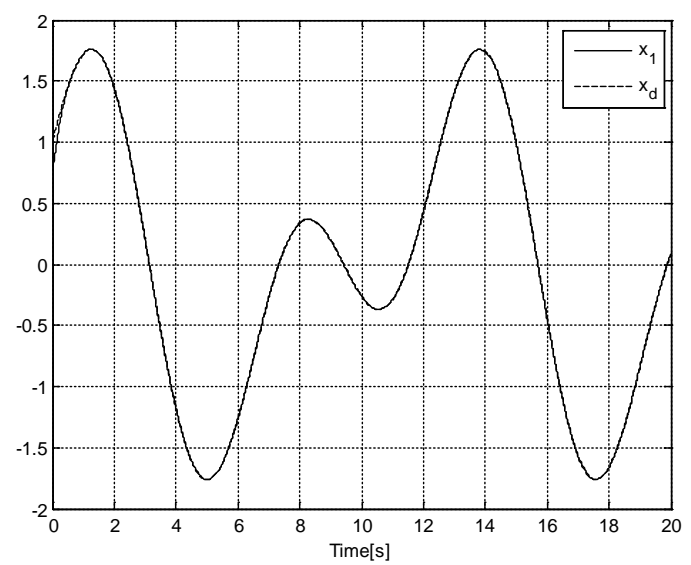

Fig. 1. System state $x_{1}$ and desired trajectory

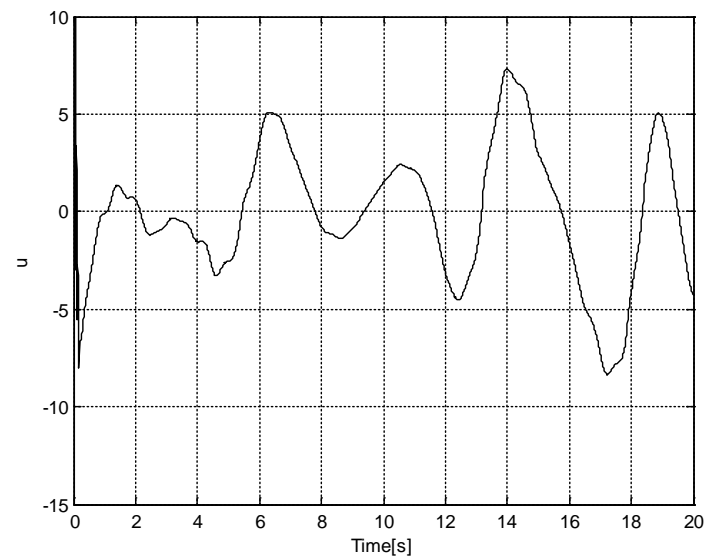

Fig. 3. Control input $u$

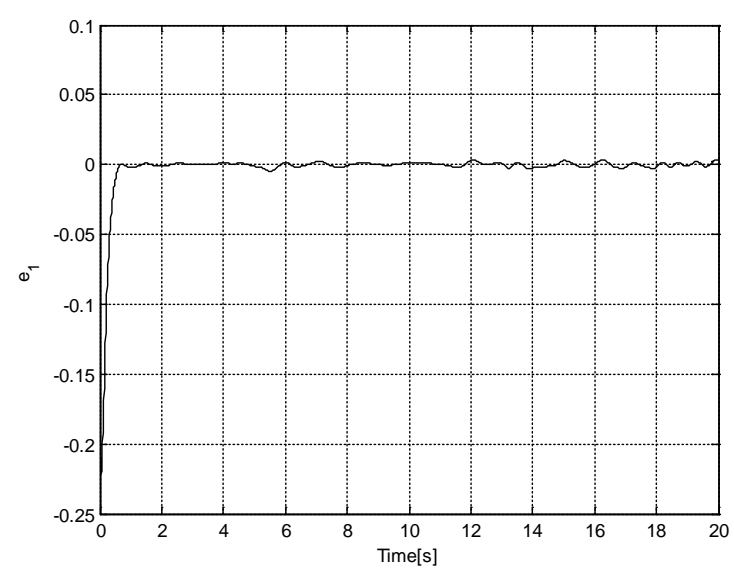

Fig. 2. Tracking error of system state $x_{1}$



Fig.4. Disturbance estimation by NDOB 


\section{Conclusion}

In this paper, the NDOB based sliding mode tracking control has been proposed for a class of uncertain nonaffine nonlinear systems. To design tracking controller, the known nonaffine nonlinear system has been transformed to the affine nonlinear system by Taylor expansion method. To improve the ability of the disturbance attenuation and system robustness, the NDOB has been used to approximate the external disturbance. Based on the estimation of NDOB, the sliding mode tracking control has been presented for the uncertain nonaffine nonlinear system with time-varying external disturbances. The stability of the closed-loop system has been proved using Lyapunov analysis. Finally, simulation results have been shown to illustrate the effectiveness of the proposed sliding mode tracking control scheme. In the future work, the developed sliding mode tracking control for the known nonaffine nonlinear systems can be extended to the unknown nonaffine nonlinear systems.

\section{Acknowledgement}

In this paper, the research was sponsored by the Nature Science Foundation of Guangdong Province (Project No. S2012040007700, S2013040012898).

\section{References}

[1] Mou Chen. Direct adaptive neural control for a class of uncertain nonaffine nonlinear systems based on disturbance observer[J]. IEEE Transactions on Cybernetics, 2013, 43(4): 1213-1225.

[2] Qiang Zhang, HongLiang Yu, Xiaohong Wang. Integral terminal sliding mode control for a class of nonaffine nonlinear systems with uncertainty[J]. Mathematical Problems in Engineering, 2013. doi:10.1155/2013/636494.

[3] Xin Zhang, Huaguang Zhang, Qiuye Sun, Yanhong Luo. Adaptive dynamic programming-based optimal control of unknown nonaffine nonlinear discrete-time systems with proof of convergence[J]. Neurocomputing, 2012, 91: 48-55.

[4] K. Ohishi, M. Nakao, K. Ohnishi, K. Miyachi. Microprocessor-controlled DC motor for load-insensitive position servo system[J]. IEEE Transactions on Industrial Electronics, 1987, 34 (1): $44-49$.

[5] Chao Liu, Jianhua Wu, Jia Liu, Zhenhua Xiong. High acceleration motion control based on a time-domain identification method and the disturbance observer[J]. Mechatronics, 2014, 24(6): 672-678.

[6] Jun Yang, Shihua Li, Xinghuo Yu. Sliding-mode control for systems with mismatched uncertainties via a disturbance observer[J]. IEEE Transactions on Industrial Elictronics, 2013, 60(1): 160-169.

[7] Divyesh Ginoya, P. D. Shendge, S. B. Phadke. Sliding mode control for mismatched uncertain systems using an extended disturbance observer[J]. IEEE Transactions on Industrial Elictronics, 2014, 61(4): 1983-1992.

[8] Lingfei Xiao, Yue Zhu. Sliding mode output feedback control based on tracking error observer with disturbance estimator[J]. ISA Transactions, 2014, 53(4): 1061-1072. 